\title{
A NEW PERTURBATION TECHNIQUE FOR DIFFERENTIAL EQUATIONS WITH SMALL PARAMETERS*
}

\author{
BY \\ M. A. BRULL AND A. I. SOLER $\dagger$ \\ University of Pennsylvania, Philadelphia, Pennsylvania
}

\begin{abstract}
Ordinary linear differential equations containing small parameter $\epsilon$ are investigated in regard to the existence of solutions in power series of the parameter. A new perturbation technique is developed which yields solutions more convenient for computation than comparable solutions obtained by making the usual series expansion in the small parameter. The new method is applied to second and fourth order differential equations in normal form and it is shown that the method yields asymptotic solution for small $\epsilon$. Conditions needed for successful application of the method are discussed and a typical solution is obtained. Comparison of numerical results with an exact solution and with an ordinary perturbation solution indicates the usefulness of the technique.
\end{abstract}

Introduction. Consider the ordinary linear differential equation

$$
L_{0}(u)+\sum_{n=1}^{\infty} \epsilon^{n} L_{n}(u)=0,
$$

where the differential operators are such that a closed form exact solution cannot be obtained or, at best, is difficult to obtain for $\epsilon \neq 0$. An approximate solution may be obtained in the form

$$
u(x)=\sum_{n=0}^{N} u_{n}(x) \epsilon^{n}, \quad N \leq \infty
$$

where $u_{0}(x)$ is the solution to (1) with $\epsilon=0$, and the successive terms $u_{n}(x)$ are obtained by solving differential equations of the type

$$
L_{0}\left(u_{n}\right)=P_{n}\left(u_{0}, u_{1}, \cdots u_{n-1}\right) .
$$

The number of terms which must be retained for numerical accuracy is strongly dependent on the value of the parameter $\epsilon$; thus, except for very small $\epsilon$, a solution of the form

$$
u(x) \approx u_{0}(x)+\epsilon u_{1}(x),
$$

truncated at the linear term in $\epsilon$ will in many cases not yield sufficient accuracy. The computation of additional terms may become exceedingly laborious, however, and the perturbation solution will lose its value as an analytical tool. In this paper, we present a new method which will, under certain conditions, increase the range of validity of perturbation methods.

We seek a new perturbation solution in the form

*Received May 14, 1965; revised manuscript received September 22, 1965.

†Formerly Member Technical Staff, Ingersoll Rand Research Center, Bedminster, N. J. 


$$
u(x)=U(x) \exp [\phi(x, \epsilon)],
$$

where $\phi(x, \epsilon)$ is a function which can be represented in the form

$$
\phi(x, \epsilon)=\sum_{n=1}^{M} \phi_{n}(x) \epsilon^{n},
$$

while $U(x)$ is the solution of (1) for $\epsilon=0$. If $\exp [\phi(x, \epsilon)]$ is expanded in a power series in $\epsilon$, then the solution (5) may be rewritten as

$$
u(x)=\sum_{n=0}^{N} \mu_{n}(x) \epsilon^{n}, \quad N \leq \infty
$$

which is the ordinary perturbation solution of (1) in the parameter $\epsilon$.

The convergence of (7) to the exact solution may be established by "term-by term" identification with the solution to the integral equation formulated from (1) by procedures similar to those set forth in $[1,2,3]$.

On the basis of the preceding remarks, it is reasonable to expect that the solution in the form of (5) (if it can be determined) could be more useful than the solution in the form of (2); the fact that the series expansion is made only on the exponent of the exponential function, rather than on the entire function, may result in a more rapidly convergent representation of the solution $u(x)$ and, in some cases, extend the range of validity of the resulting solutions.

Perturbation Method for Ordinary Differential Equations of Second Order. In this section we consider the general second-order linear differential equation and develop a new method for obtaining a perturbation solution. The differential equation may be written in the normal form:

$$
u^{\prime \prime}+f(x, \epsilon) u=0,
$$

where $f(x, \epsilon)$ is assumed of the form

$$
f(x, \epsilon)=g_{0}(x)+G(x, \epsilon)
$$

and $G(x, \epsilon)$ satisfies the condition

$$
G(x, 0)=0 .
$$

It is, of course, implied that the solution of the differential (8) for $\epsilon=0$ is known and will be used as the starting point for the perturbation as in the usual technique.

We now seek a particular solution in the form,

$$
u(x)=w(x) \exp [\phi(x, \epsilon)],
$$

where $w(x)$ and $\phi(x, \epsilon)$ are functions to be determined. Substituting (9) and (11) into (8) gives

$$
w^{\prime \prime}+g_{0}(x) w+2 w^{\prime} \phi^{\prime}+w \phi^{\prime \prime}+w{\phi^{\prime}}^{2}+G(x, \epsilon)=0 .
$$

We note that the first two terms of (12) are independent of $\epsilon$ and that their sum must, therefore, be set equal to zero since the equation must be satisfied for all values of $\epsilon$. We, therefore, obtain from (12) the following two equations:

$$
\begin{aligned}
& w^{\prime \prime}+g_{0}(x) w=0, \\
& \phi^{\prime \prime}+2 \frac{w^{\prime}}{w} \phi^{\prime}+{\phi^{\prime}}^{2}=-G(x, \epsilon) .
\end{aligned}
$$


We note from (13) that $w(x)$ is now identified as the starting solution of (8) for $\epsilon=0$ and it follows that

$$
\phi(x, 0)=0 .
$$

We also note that, since $\phi$ does not appear explicitly in Eq. (14), we may write this equation in the form,

$$
H^{\prime}+2 \frac{w^{\prime}}{w} H+H^{2}=-G(x, \epsilon)
$$

where

$$
H(x, \epsilon)=\phi^{\prime}(x, \epsilon) .
$$

At this point we have transformed our second-order linear differential equation into a non-linear first-order equation of the Ricatti type. The similarity of the technique up to this point with both the $W K B J$ method and the classical method of asymptotic integration will be noted. We now investigate the existence of a perturbation solution of (16) valid for small $\epsilon$. To this end we expand $G(x, \epsilon)$ in a power series in $\epsilon$ of the form,

$$
G(x, \epsilon)=\sum_{n=1}^{\infty} g_{n}(x) \epsilon^{n},
$$

where the leading term is linear in $\epsilon$ by virtue of (10). We seek for $H(x, \epsilon)$ a solution in the form

$$
H(x, \epsilon)=\sum_{n=1}^{\infty} h_{n}(x) \epsilon^{n} .
$$

Substituting (18) into (16), expanding the non-linear term by means of the Cauchy product, and remembering that $h_{0}(x)=0$, we obtain, after equating coefficients of like powers of $\epsilon$, the following differential equation for the coefficients $h_{n}(x)$.

$$
h_{n}^{\prime}+\frac{2 w^{\prime}}{w} h_{n}(x)=-g_{n}(x)-\sum_{k=1}^{n-1} h_{k}(x) h_{n-k}(x) ; \quad n=1,2, \cdots
$$

A solution of (19) is easily obtained as,

$$
h_{n}(x)=-\frac{1}{w^{2}} \int\left[g_{n}(x)+\sum_{k=1}^{n-1} h_{k}(x) h_{n-k}(x)\right] w^{2}(x) d x .
$$

It is clear from (11) and (18) that the complete perturbation solution of (8) obtained in this manner is of the form,

$$
u(x)=A_{1} w_{1}(x) \exp \left[\sum_{n=1}^{\infty} \epsilon^{n} \int h_{n 1}(x) d x\right]+A_{2} w_{2}(x) \exp \left[\sum_{n=1}^{\infty} \epsilon^{n} \int h_{n 2}(x) d x\right],
$$

where $w_{i}(x) i=1,2$ are the independent solutions of (13) and $h_{n i}(x) i=1,2$ are the functions obtained from (20) using the appropriate $w_{i}(x)$.

We will now show that (21) is in fact either an asymptotic representation of $u(x)$ for small values of the parameter $\epsilon$ or a convergent series representation of $u(x)$. To this end, consider the $m$ th partial sum of either of the independent perturbation series solutions in (21) which may be written as, 


$$
u_{m}(x)=w(x) \exp \left[\sum_{n=1}^{m} \epsilon^{n} \int h_{n}(x) d x\right] .
$$

We now define as $\eta_{m}(x)$ the residual due to substituting $u_{m}(x)$ for $u(x)$ in (8). Substituting (22) into (8), and making use of (9) and (17), we obtain, after rearranging,

$$
\eta_{n_{\iota}}(x)=\sum_{n=1}^{m} w\left[h_{n}^{\prime}+2 \frac{w^{\prime}}{w} h_{n}+\sum_{k=1}^{n-1}\left(h_{k} h_{n-k}\right)+g_{n}\right] \epsilon^{n}+\sum_{n=m+1}^{\infty} g_{n}(x) \epsilon^{n} .
$$

We note that the coefficient of $\epsilon^{n}$ in the first scries of (23) vanishes by virtue of (19). Thus, we may write the residual in the form

$$
\eta_{m}(x)=\sum_{n=m+1}^{\infty} g_{n}(x) \epsilon^{n}
$$

We may, therefore, write the equation

$$
\left(\frac{1}{\epsilon}\right)^{m} \eta_{m}(x)=\sum_{r=1}^{\infty} g_{m+r}(x) \epsilon^{r} .
$$

Equation (25) now reveals the true nature of the perturbation solution; we note that $\eta_{m}(x)$ satisfies the condition

$$
\lim _{\substack{\epsilon \rightarrow 0 \\ m \text { fixed }}}\left(\frac{1}{\epsilon}\right)^{m}\left|\eta_{m}(x)\right|=0
$$

because the lowest power term in the series of (25) is always at least linear in $\epsilon$. Thus, we can state that the differential equation is always satisfied asymptotically for small $\epsilon$ by the perturbation solution of $(21)$. Since $\eta_{m}(x)$ was obtained applying to $u_{n}(x)$ defined by (22) a sequence of linear operations, we conclude that $u_{m}(x)$ is an asymptotic representation of the solution $u(x)$ of our differential equation. If it can be proved in any specific case that the series in (21) converges, then the solution is exact rather than asymptotic. Thus, we have proved that as long as the function $G(x, \epsilon)$ admits the representation (17) the perturbation method will yield either an asymptotic solution for small $\epsilon$ or an exact series representation in $\epsilon$ of the solution. It is clear, therefore, that $G(x, \epsilon)$ must be an analytic function of $\epsilon$, in order for the method to apply. It should be noted that in many instances where convergence of the solution can be established, the present solution will converge faster than the ordinary perturbation solution. Furthermore, in cases when the classical perturbation method fails, the present method may yield an asymptotic representation suitable for computation. Thus the range of $\epsilon$ over which a perturbation technique may be utilized will be extended.

Application of the Technique. The applicability of the new technique is illustrated by considering a differential equation with variable coefficients the exact solution of which is obtainable in closed form. Perturbation solutions are obtained using the new technique and the classical power series expansion in terms of the small parameter; comparison of results indicates the greater range of validity of the new technique for the same number of terms.

We consider an equation from the theory of symmetrically loaded circular plates with variable flexural rigidity. In terms of suitably defined dimensionless variables, the equation to be studied may be written in the form [4]: 


$$
W^{\prime \prime}+\left[\frac{1}{x}+(\ln D)^{\prime}\right] W^{\prime}+\left[\frac{v}{x}(\ln D)^{\prime}-\frac{1}{x^{2}}\right] W=0 .
$$

Defining the new dependent variable $w(x)$ such that

$$
W(x)=w(x) /(x D)^{1 / 2},
$$

yields the normal form of (27) as,

$$
w^{\prime \prime}(x)+\left[-\frac{3}{4 x^{2}}-\frac{1}{2}(\ln D)^{\prime \prime}-\frac{(1-2 v)}{2 x}(\ln D)^{\prime}-\left(\frac{1}{2}[\ln D]^{\prime}\right)^{2}\right] w(x)=0 .
$$

If the flexural rigidity is assumed to vary according to the expression:

$$
\ln D / D_{0}=\epsilon \int f(\rho) d \rho
$$

(29) has the form of (8) if

$$
\begin{array}{ll}
g_{0}(x)=-\frac{3}{4 x^{2}}, & g_{1}(x)=-\left[\frac{1}{2} f^{\prime}+\frac{(1-2 v)}{2 x} f\right], \\
g_{2}(x)=-\frac{1}{4} f^{2}(x), & g_{n}(x)=0 . \quad n>2
\end{array}
$$

For the stiffness distribution

$$
f(x)=1 / x
$$

the exact solution of (27) is given by

where

$$
\begin{aligned}
W(x) & =A x^{-(\alpha+\epsilon / 2)}+B x^{(\alpha-\epsilon / 2)}, \\
\alpha & =\left[1-\nu \epsilon+\epsilon^{2} / 4\right]^{\frac{1}{3}} .
\end{aligned}
$$

Substituting the first equation of (31) into (13) yields the two independent starting solutions

$$
w_{1}(x)=x^{3}, \quad w_{2}(x)=x^{-\frac{1}{2}},
$$

and hence (29) yields

$$
\begin{array}{ll}
h_{11}(x)=-\nu / 2 x ; & h_{21}=\nu / 2 x \\
h_{22}(x)=\left(1-\nu^{2}\right) / 8 x ; & h_{22}=-\left(1-\nu^{2}\right) / 8 x
\end{array}
$$

Substituting these results into (21) and using (28) results in an approximate solution to (27) (which satisfies the differential equation up to and including terms of order $\epsilon^{2}$ ) in the form

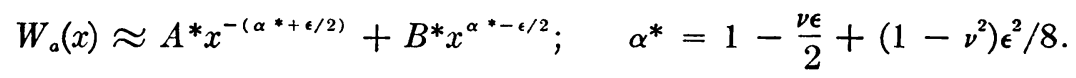

The classical perturbation solution for $W(x)$ containing second order correction terms admits the representation

$$
(x D)^{\frac{1}{2}} W_{c}(x) \approx C_{1}\left(u_{10}+\epsilon u_{11}+\epsilon^{2} u_{12}\right)+C_{2}\left(u_{20}+\epsilon u_{22}+\epsilon^{2} u_{22}\right)
$$

where the governing equations for determining $u_{i j}(x)$ are obtained by substituting (37) into (29) and equating terms in like powers of $\epsilon$. The resulting perturbation equations 
yield the starting solutions

$$
u_{10}(x)=x^{\frac{3}{2}}, \quad u_{20}(x)=x^{-\frac{1}{2}},
$$

and the differential equations

$$
\left.\begin{array}{l}
u_{i 1}^{\prime \prime}-\frac{3}{4 x^{2}} u_{i 1}=-\left(\frac{\nu}{x^{2}}\right) u_{i 0}(x), \\
u_{i 2}^{\prime \prime}-\frac{3}{4 x^{2}} u_{i 2}=-\left(\frac{\nu}{x^{2}}\right) u_{i 1}(x)+\frac{u_{i n} .}{4 x^{2}} .
\end{array}\right\}
$$

These equations give the perturbation solutions which may be substituted into (37) to yield the power series solution

$$
\begin{aligned}
(x D)^{\frac{3}{3}} W_{c}(x) \approx C_{1} x^{\frac{3}{2}}[1 & \left.-\left(\frac{\nu \epsilon}{2}-\epsilon^{2}\left[1-\nu^{2}\right] / 8\right) \ln x+\left(\nu^{2} \epsilon^{2} / 8\right) \ln ^{2} x\right] \\
& +C_{2} x^{-\frac{1}{2}}\left[1+\left(\frac{\nu \epsilon}{2}-\epsilon^{2}\left[1-\nu^{2}\right] / 8\right) \ln x+\left(\nu^{2} \epsilon^{2} / 8\right) \ln ^{2} x\right] .
\end{aligned}
$$

It can easily be shown that if (36) is expanded in ascending powers of $\epsilon$ by writing

$$
x^{\beta}=\exp [\beta \ln x]=1+\beta \ln x+\cdots .
$$

then the resulting expansion (keeping only second order terms in $\epsilon$ ) is identical to (40). Hence, for this case, the new perturbation technique can be thought of as being obtained by recognizing certain groupings of terms in the classical series expansion as being expressible in closed form.

As a numerical example, consider the boundary conditions

$$
W^{\prime}\left(x_{0}\right)=0, \quad W(1)=V .
$$

The exact solution and the two approximate solutions yield the following representations in the range $x_{0} \leq x \leq 1$ :

Exact Solution:

$$
\frac{W(x)}{V}=\frac{x^{\alpha-\epsilon / 2}}{1+\frac{\alpha-\epsilon / 2}{\alpha+\epsilon / 2} x_{0}^{2 \alpha}}\left[1+\frac{\alpha-\epsilon / 2}{\alpha+\epsilon / 2}\left(\frac{x_{0}}{x}\right)^{2 \alpha}\right] .
$$

New Perturbation Technique:

$$
\frac{W_{a}(x)}{V}=\frac{x^{\alpha^{*-\epsilon / 2}}}{1+\frac{\alpha^{*}-\epsilon / 2}{\alpha^{*}+\epsilon / 2} x_{0}^{2 \alpha *}}\left[1+\frac{\alpha^{*}-\epsilon / 2}{\alpha^{*}+\epsilon / 2}\left(\frac{x_{0}}{x}\right)^{2 \alpha^{*}}\right] .
$$

Classical Perturbation Technique:

$$
\begin{aligned}
\frac{W_{c}(x)}{V}=\frac{\eta}{\eta-\mu} & x^{-(1+\epsilon / 2)}\left[1+\frac{\epsilon}{2}\left(\nu-\epsilon\left[1-\nu^{2}\right] / 4\right) \ln x+\left(\nu^{2} \epsilon^{2} / 8\right) \ln ^{2} x\right] \\
-\frac{\mu}{\eta-\mu} x^{(1-\epsilon / 2)} & {\left[1-\frac{\epsilon}{2}\left(\nu-\epsilon\left[1-\nu^{2}\right] / 4\right) \ln x+\left(\nu^{2} \epsilon^{2} / 8\right) \ln ^{2} x\right], }
\end{aligned}
$$

where $\eta$ and $\mu$ are defined by 


$$
\begin{aligned}
& \eta \approx x_{0}^{-\epsilon / 2}\left\{\left[1-\frac{\epsilon(1+\nu)}{2}+\frac{\epsilon^{2}\left(1-\nu^{2}\right)}{8}\right]\right. \\
& \left.+\left[\frac{\nu \epsilon}{2}\left(\frac{\epsilon}{2}-1\right)+\frac{\epsilon^{2}\left(1+\nu^{2}\right)}{8}\right] \ln x_{0}+\frac{\nu^{2} \epsilon^{2}}{8} \ln ^{2} x_{0}\right\}, \\
& \mu \approx-x_{0}^{-(2+\epsilon / 2)}\left\{\left[1+\frac{\epsilon(1-\nu)}{2}+\frac{\epsilon^{2}\left(1-\nu^{2}\right)}{8}\right]\right. \\
& \left.+\left[\frac{\nu \epsilon}{2}\left(\frac{\epsilon}{2}+1\right)-\frac{\epsilon^{2}\left(1+\nu^{2}\right)}{8}\right] \ln x_{0}+\frac{\nu^{2} \epsilon^{2}}{8} \ln ^{2} x_{0}\right\} .
\end{aligned}
$$

\begin{tabular}{|c|c|c|c|c|c|}
\hline$\epsilon$ & $W / V$ & $W_{a} / V$ & $E_{a}(\%)$ & $W_{c} / V$ & $E_{c}(\%)$ \\
\hline .1 & .4055 & .4070 & -.370 & .4073 & $-\quad .444$ \\
\hline 1.0 & .6060 & .6140 & -1.320 & .6062 & $-\quad .033$ \\
\hline 2.0 & .7490 & .7820 & -4.400 & .4920 & +34.300 \\
\hline 3.0 & .9483 & .8933 & +5.740 & -2.080 & +320.000 \\
\hline 4.0 & 1.781 & .7375 & +58.500 & $\begin{array}{l}\text { not com- } \\
\text { puted }\end{array}$ & - \\
\hline
\end{tabular}

Numerical results are computed at $x=x_{0}=0.2$ for various values of $\epsilon$ and the results are presented in Table 1 . where $E_{i}(i=a, c)$ is a measure of the error incurred by using

TABLE $1-$ Numerical results for $x=x_{0}=0.2 ; \nu=.3$

perturbation solutions up to second power terms:

$$
E_{i}=\frac{W-W_{i}}{W} \times 100 \% .
$$

For the selected problem, the new perturbation technique yields results for the displacement which compare well with the exact solution over a wider range of $\epsilon$ than the classical perturbation method. In addition, a comparison of the labor involved to obtain (36) and (40) reveals that the new technique requires much less effort to obtain higher approximations (these additional terms being easily computed by repeated application of (20)); the resulting expressions are also much more convenient for hand computation.

Perturbation Method for Ordinary Differential Equations of Fourth Order. The development of the new perturbation method given in detail in the previous section for second-order differential equations may easily be extended to fourth-order differential equations. This will be outlined in this section and only essential results will be shown in the interest of brevity.

We consider the most general linear differential equation of fourth-order in the form,

$$
w^{\prime v}+A(x, \epsilon) w^{\prime \prime}+B(x, \epsilon) w^{\prime}+C(x, \epsilon) w=0 .
$$

It should be noted that a suitable transformation of the dependent variable can always remove the third derivative term from a fourth-order linear differential equation so that (48) does indeed represent the most general form. The coefficient functions $A(x, \epsilon)$, $B(x, \epsilon)$ and $C(x, \epsilon)$ are, as before, assumed to be analytic functions of $\epsilon$ and therefore admit MacLaurin series expansions: 


$$
\begin{aligned}
& A(x, \epsilon)=\sum_{n=0}^{\infty} a_{n}(x) \epsilon^{n}, \\
& B(x, \epsilon)=\sum_{n=0}^{\infty} b_{n}(x) \epsilon^{n}, \\
& C(x, \epsilon)=\sum_{n=0}^{\infty} c_{n}(x) \epsilon^{n} .
\end{aligned}
$$

We again seek a solution in the form,

$$
w(x, \epsilon)=u(x) e^{\phi(x, \epsilon)},
$$

where $u(x)$ represents one of the independent solutions of (48) for $\epsilon=0$ and $w(x)$ denotes the corresponding solution for $\epsilon \neq 0$. When (50) is substituted into (48), there is obtained a linear differential equation for $u(x)$ and a non-linear differential equation for $\phi(x, \epsilon)$. The equation for $u(x)$ is:

$$
u^{\mathrm{iv}}+a_{0}(x) u^{\prime \prime}+b_{0}(x) u^{\prime}+C_{0}(x) u=0
$$

which shows that $u(x)$ is indeed a solution of (48) corresponding to $\epsilon=0$. The nonlinear equation for $\phi$ may be written in the form

$$
\begin{aligned}
& u \phi^{\mathrm{iv}}+4 u^{\prime} \phi^{\prime \prime \prime}+\left[6 u^{\prime \prime}+a_{0} u\right] \phi^{\prime \prime}+\left[4 u^{\prime \prime \prime}+2 a_{0} u^{\prime}+b_{0} u\right] \phi^{\prime}+[A(x, \epsilon) \\
& \left.-a_{0}(x)\right]\left[2 u^{\prime} \phi^{\prime}+u \phi^{\prime \prime}+u \phi^{\prime 2}+u^{\prime \prime}\right]+\left[B(x, \epsilon)-b_{0}(x)\right]\left[u \phi^{\prime}+u^{\prime}\right] \\
& +\left[C(x, \epsilon)-c_{0}(x)\right] u+3 u \phi^{\prime \prime 2}+4 u \phi^{\prime} \phi^{\prime \prime \prime}+6 u^{\prime \prime}{\phi^{\prime}}^{2}+a_{0} u{\phi^{\prime}}^{2}+12 u^{\prime} \phi^{\prime} \phi^{\prime \prime} \\
& +6 u{\phi^{\prime 2}}^{2} \phi^{\prime \prime}+4 u^{\prime} \phi^{\prime 3}+u \phi^{\prime 4}=0 \text {. }
\end{aligned}
$$

Following the method of the previous section, we seek a solution of (52) in the form,

$$
\phi(x, \epsilon)=\sum_{n=1}^{\infty} \psi_{n}(x) \epsilon^{n} .
$$

Substituting (49) and (53) into (52), making use of the Cauchy product to calculate non-linear terms and equating coefficients if like powers of $\epsilon$, we obtain a general recurrence relation for $\psi_{n}(x)$. The result is

$$
\begin{aligned}
\psi_{n}^{\text {iv }}+4 \frac{u^{\prime}}{u} \psi_{n}^{\prime \prime \prime}+\left(6 \frac{u^{\prime \prime}}{u}\right. & \left.+a_{0}\right) \psi_{n}^{\prime \prime}+\left(4 \frac{u^{\prime \prime \prime}}{u}+2 a_{0} \frac{u^{\prime}}{u}+b_{0}\right) \psi_{n}^{\prime} \\
& =-\left[a_{n} \frac{u^{\prime \prime}}{u}+b_{n} \frac{u^{\prime}}{u}+c_{n}\right]+F_{n}(x), \quad n=1,2 \cdots
\end{aligned}
$$

where

$$
\begin{aligned}
F_{n}(x)=-\sum_{k=1}^{n-1}\left[2 \frac{u^{\prime}}{u} a_{k} \psi_{n-k}^{\prime}+a_{k} \psi_{n-k}^{\prime \prime}+b_{k} \psi_{n-k}^{\prime}+3 \psi_{k}^{\prime \prime} \psi_{n-k}^{\prime \prime}+4 \psi_{k}^{\prime} \psi_{n-k}^{\prime \prime \prime}\right. \\
\left.+6 \frac{u^{\prime}}{u} \psi_{k}^{\prime} \psi_{n-k}^{\prime}+a_{0} \psi_{k}^{\prime} \psi_{n-k}^{\prime}+12 \frac{u^{\prime}}{u} \psi_{k}^{\prime} \psi_{n-k}^{\prime \prime}\right] \\
-\sum_{k=1}^{n-1} \sum_{r=1}^{k-1}\left[6 \psi_{n-k}^{\prime \prime}+4 \frac{u^{\prime}}{u} \psi_{n-k}^{\prime}+a_{n-k}\right] \psi_{r}^{\prime} \psi_{k-r}^{\prime} \\
-\sum_{k=1}^{n-1} \sum_{r=1}^{k-1} \sum_{i=1}^{n-k-1} \psi_{r}^{\prime} \psi_{k-r}^{\prime} \psi_{s}^{\prime} \psi_{n-k-s}^{\prime}
\end{aligned}
$$


$\psi_{0}(x)$ is taken as zero in accordance with (50) and (53). We note that (54) is fairly complex and will generally have variable coefficients unless (51) has constant coefficients and single fold characteristic roots so that $u(x)=e^{\lambda x}$. It should be noted that if (51) has constant coefficients but multiple fold characteristic roots, one of the independent solutions for $U$ will be of the form $u(x)=x^{p} e^{\lambda x}$ and the corresponding equation (54) will have variable coefficients. It is clear, however, that when (51) has constant coefficients and single fold characteristic roots, (54) has constant coefficients, and the desired particular integral of (54) is easy to determine. The only limitation on the application of the method is then the increasing complexity of $F_{n}(x)$ as $n$ increases.

It should be noted that a solution of the form (50) must be obtained for each independent solution of (51).

By carrying out a proof similar to that of the preceding section, it can be shown that the solution (50) satisfies (48) asymptotically for small $\epsilon$ and is therefore an asymptotic representation of $w(x, \epsilon)$ valid for small $\epsilon$. As before, if the convergence of the series (53) can be established, the solution (50) becomes exact rather than asymptotic.

Conclusions. A new perturbation technique for linear differential equations with a small parameter has been developed and leads to solutions which are in many cases more convenient for computation than those obtained by the ordinary perturbation technique. It has been shown that, the new method yields an asymptotic representation of the solution for small values of the parameter. The theory is presented in general form for second and fourth order linear differential equations so that the reader need not repeat the substitutions into the original differential equations but can proceed directly to the determination of the perturbation solution for any particular equation.

The numerical example presented in the paper indicates a substantial increase in accuracy over the classical perturbation method as well as an extension in the range of values of the parameter for which the perturbation method can be utilized.

\section{REFERENCES}

1. J. R. Vinson and M. A. Brull, New Techniques of Solutions for Problems in the Theory of Ortholropic Plates, Proc. 4th U. S. National Congress of Applied Mechanics, p. 817 (1962)

2. A. I. Soler, Application of Perturbation Techniques to the Solution of Problems in Thermoviscoelasticity, Ph.D. Thesis, University of Pennsylvania, December, (1962)

3. A. I. Soler and M. A. Brull, On the Solution to Transient Coupled Thermoelastic Problems by Perturbation Techniques, Jour. of Applied Mech., 32, no. 2, p. 384, June (1965)

4. E. H. Mansfield, The Bending and Stretching of Plates, MacMillan, New York, p. 77 (1964) 